\title{
Hybrid Link Adaptation for ICI Adjustment in OFDMA Cellular Uplink Systems
}

\author{
Jin-Woo Lee and Yong-Hwan Lee \\ School of Electrical Engineering and INMC \\ Seoul National University \\ Kwanak P. O. Box 34, Seoul 151-600 Korea \\ E-mail:jinu@ttl.snu.ac.kr,ylee@snu.ac.kr
}

\begin{abstract}
In orthogonal frequency division multiple access (OFDMA)-based uplink systems, it may not be feasible to exploit the rate adaptation due to severe intercarrier interference (ICI). In this paper, we propose a novel hybrid link adaptation scheme that employs power adaptation for ICI adjustment and rate adaptation for capacity improvement in OFDMAbased uplink systems. The proposed scheme determines the maximum allowable transmit power in response to the channel gain and mobility of the user, guaranteeing the carrier to interference power ratio (CIR) loss of all users.
\end{abstract}

\section{Introduction}

In cellular communication systems, the quality of received signal is severely affected by the operating environments. It is desirable to accommodate the signal transmission according to the variation of signal quality. To this end, conventional wireless systems employ a so-called link adaptation technique that often adjusts the transmit power or the transmission rate [1].

The power adaptation technique adjusts the transmit power to compensate for the attenuation of channel gain $[2,3]$. It usually reduces the transmit power when the user is close to the base station (BS) and increases it when the user is far away from the BS. It has been applied to the $2 \mathrm{G}$ and $3 \mathrm{G}$ code division multiple access (CDMA) systems to compensate for inter-cell interference in the downlink and to reduce the intracell interference (near-far problem) in the uplink. On the other hand, the rate adaptation technique adjusts the modulation parameters (e.g., the modulation level and code rate) according to the quality of the received signal, while keeping the transmit power unchanged [4-6]. It often transmits the signal using a high order

This work was in part supported by Samsung Eletronics, Inc. modulation with high code rates when the user is close to the BS, and vice versa.

Recently, orthogonal frequency division multiple access (OFDMA) systems have attracted much attention as a key transmission technique for next generation (so called 4G) wireless access systems [79]. In the downlink of OFDMA systems, the rate adaptation technique is mainly employed to improve the system capacity $[10,11]$. However, when applied to the uplink, it may cause a serious inter-carrier interference (ICI) problem. Each user is only affected by its own ICI in the downlink [12], but it can be affected by ICI from other users in the uplink. Thus, the user near the BS and/or with high mobility can cause severe ICI effect to other users in the uplink. In the uplink, conventional OFDMA systems consider to simply adjust the transmit power to alleviate severe ICI problem $[10,11]$. They adjust the transmit power to limit the ICI only in response to the user channel gain regardless of the user mobility. However, they may not provide optimum performance in view of the system capacity. Users with low mobility near the cell boundary may not need to adjust the power, rather need to adjust the rate to lower the amount of ICI. It was shown that the use of power adaptation may not provide the improvement of system capacity over the use of rate adaptation $[1,13]$.

In this paper, we consider a novel hybrid link adaptation scheme that adjusts the transmit power to limit the ICI, in addition to the adaptation of transmission rate to improve the system capacity in the uplink of OFDMA cellular systems. The proposed scheme determines the maximum allowable transmit power in response to the user channel gain and mobility. As a result, it can provide higher system capacity compared to the use of pure power adaptation by applying the rate adaptation to users with low mobility near the cell boundary, while providing all users with a desired loss of the carrier to interference power ratio (CIR). 


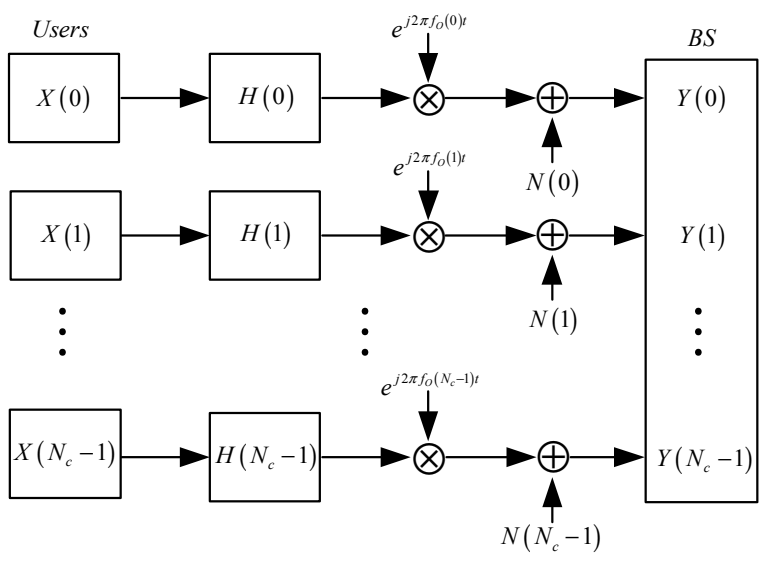

Figure 1. OFDMA uplink system model.

The rest of this paper organized as follows. Section II describes the ICI model in OFDMA uplink. In Section III, we propose a novel hybrid link adaptation scheme for the adjustment of ICI. The performance of the proposed scheme is verified by computer simulation in Section IV. Finally, conclusions are summarized in Section V.

\section{Inter-carrier interference in the uplink}

We consider the uplink of a multi-user OFDMA wireless system, where the whole bandwidth $B$ is partitioned into $N_{c}$ number of subcarriers. Figure 1 shows a simplified block diagram of the OFDMA uplink system in consideration. The user symbol allocated at the $i$-th subcarrier $X(i)$ is transmitted through the corresponding sub-channel $H(i)$. The received signal may experience frequency offset by an amount of $f_{o}(i)$ due to Doppler frequency $f_{d}(i)$ and oscillator frequency offset $f_{o}(i)$ of the user as

$$
f_{o}(i)=f_{d}(i)+f_{o}(i)
$$

where $f_{d}(i) \simeq f_{c} v(i) / c$. Here, $f_{c}$ denotes the carrier frequency, and $c$ and $v(i)$ denote the velocity of light and user allocated at the $i$-th subcarrier, respectively. In the presence of additive noise $N(i)$, the received signal $Y(i)$ can be represented as

$$
Y(i)=H(i) X(i) \operatorname{sinc}\left(f_{O}(i) T\right)+N(i)
$$

where $T$ denotes the OFDM symbol duration and $\operatorname{sinc}(x)=\sin (\pi x) / \pi x$.

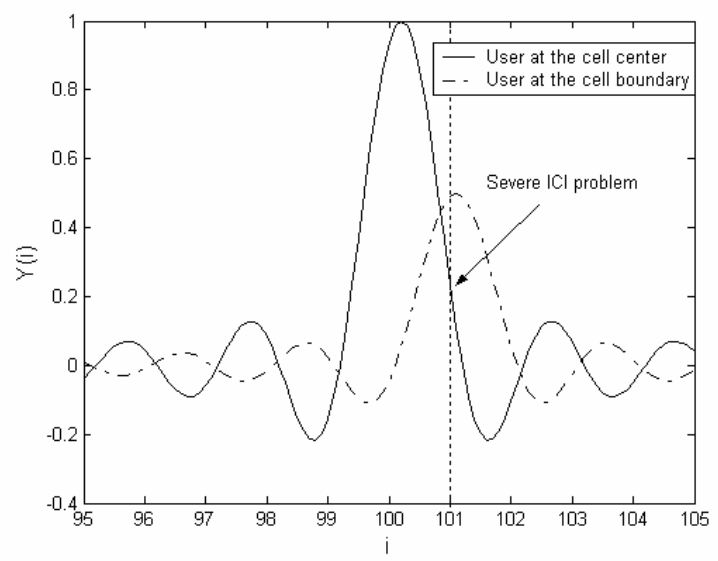

Figure 2. ICI in OFDMA uplink systems.

The ICI power from the user signal allocated at the $i$-th subcarreir to other user allocated at the $j$-th subcarrier can be represented as

$$
\begin{aligned}
P_{I C I}(i, j) & =|H(i)|^{2} P_{X}(i) \operatorname{sinc}^{2}\left(i-j+f_{O}(i) T\right) \\
& \simeq|H(i)|^{2} P_{X}(i) \operatorname{sinc}^{2}\left(i-j+\left(f_{c} \frac{v(i)}{c}+f_{o}(i)\right) T\right)
\end{aligned}
$$

where $P_{X}(i)$ is the transmit power of the user allocated at the $i$-th subcarrier (i. e., $P_{X}(i)=|X(i)|^{2}$ ). It can be seen that the ICI power is a function of the user channel gain, mobility and transmit power.

\section{Proposed link adaptation scheme}

Unlike in the downlink, it may not be feasible to employ a rate adaptation scheme without power adjustment in the uplink due to serious ICI problem. As illustrated in Figure 2, the user near the cell boundary may experience high CIR loss due to the ICI from fast users near the cell center. Consequently, the quality of the signal transmitted near the cell boundary may not be guaranteed in the uplink environments. To alleviate this problem, conventional OFDMA systems consider the use of power adaptation to compensate for large signal attenuation in the uplink $[10,11]$. However, the use of power adaptation may not provide capacity improvement over the use of rate adaptation $[1,13]$. Thus, it may not be desirable to apply a power adaptation scheme to users with low mobility in view of the system capacity.

To mitigate the ICI problem, we consider to limit the maximum allowable transmit power in an analytic manner. Since the ICI power depends on the user channel gain, mobility and transmit power, its 
maximum level should be limited to a desired level

$$
\begin{aligned}
& P_{I C I_{-} \max }, \text { i.e., } \\
& \forall i \in\left[0, N_{c}-1\right] \\
& P_{I C I}(i)=\sum_{\substack{j=0 \\
j \neq i}}^{N_{c}-1}|H(i)|^{2} P_{X}(i) \operatorname{sinc}^{2}\left(i-j+f_{O}(i) T\right) \leq P_{I C I_{-} \max } .
\end{aligned}
$$

Since the total amount of ICI power on the user allocated to the $j$-th subcarrier is

$$
P_{I C I}^{\prime}(j)=\sum_{\substack{i=0 \\ i \neq j}}^{N_{c}-1}|H(i)|^{2} P_{X}(i) \operatorname{sinc}^{2}\left(i-j+f_{O}(i) T\right),
$$

the CIR loss due to the ICI of the user allocated to the $j$-th subcarrier can be represented as

$$
\begin{aligned}
\Delta \gamma(j) & =\gamma(j)-10 \log _{10} \frac{|H(j)|^{2} P_{X}(j)}{P_{N}(j)+P_{I C I}^{\prime}(j)} \\
& =10 \log _{10}\left[1+\frac{P_{I C I}^{\prime}(j)}{|H(j)|^{2} P_{X}(j)} 10^{\frac{\gamma(j)}{10}}\right]
\end{aligned}
$$

where $P_{N}(j)$ denotes the noise power and $\gamma(j)$ denotes the CIR of the user allocated to the $j$-th subcarrier in the absence of ICI, represented as

$$
\gamma(j)=10 \log _{10} \frac{|H(j)|^{2} P_{X}(j)}{P_{N}(j)} .
$$

Note that $\Delta \gamma(j)$ and $\gamma(j)$ are represented in a decibel (dB) scale.

We assume that the CIR loss should be less than a desired value $\Delta \gamma_{\max }[14]$

$$
\Delta \gamma(j) \leq \Delta \gamma_{\max }, \forall j \in\left[0, N_{c}-1\right] .
$$

Letting

$$
P_{I C I}(i)=P_{I C I_{-} \max }, \forall i \in\left[0, N_{c}-1\right], i \neq j
$$

as the worst case to the user allocated to the $j$-th subcarrier, it can be shown that, unless $N_{c}$ is too small

$$
P_{I C I}^{\prime}(j) \approx P_{I C I}(i)=P_{I C I_{-} \max } .
$$

Then, the user allocated to the $j$-th subcarrier experiences a maximum CIR loss given by

$$
\begin{aligned}
\Delta \gamma_{\max }=10 \log _{10}\left[1+\frac{P_{I C I}(i)}{|H(j)|^{2} P_{X}(j)} 10^{\frac{\gamma(j)}{10}}\right] \\
=10 \log _{10}\left[\begin{array}{l}
1+\frac{|H(i)|^{2} P_{X}(i)}{|H(j)|^{2} P_{X}(j)} \\
\times\left\{\sum_{\substack{j=0 \\
j \neq i}}^{N_{c}-1} \operatorname{sinc}^{2}\left(i-j+f_{O}(i) T\right)\right\} 10^{\frac{\gamma(j)}{10}}
\end{array}\right] .
\end{aligned}
$$

Assuming that the noise power of each subcarrier is the same (i.e., $\left.P_{N}(j)=P_{N}\right)$, (11) can be simplified to

$$
\Delta \gamma_{\max }=10 \log _{10}\left[1+\left\{\sum_{\substack{j=0 \\ j \neq i}}^{N_{c}-1} \operatorname{sinc}^{2}\left(i-j+f_{O}(i) T\right)\right\} 10^{\frac{\gamma(i)_{\max }}{10}}\right]
$$

where $\gamma(i)_{\max }$ is the maximum CIR of the user allocated to the $i$-th subcarrier. For a large $N_{c}, \Delta \gamma_{\max }$ can be approximated as [12]

$$
\Delta \gamma_{\max } \approx 10 \log _{10}\left[1+\left\{1-\operatorname{sinc}^{2}\left(f_{O}(i) T\right)\right\} 10^{\frac{\gamma(i)_{\max }}{10}}\right] .
$$

Using a Taylor series expansion in the presence of a small frequency offset (i.e., $\operatorname{sinc}^{2}(\varepsilon) \simeq 1-(\pi \varepsilon)^{2} / 3$ ), (13) can further be approximated as

$$
\Delta \gamma_{\max } \approx 10 \log _{10}\left[1+\frac{\pi^{2}}{3}\left(f_{O}(i) T\right)^{2} 10^{\frac{\gamma(i)_{\max }}{10}}\right] .
$$

Or, $\gamma(i)_{\max }$ can be represented as

$$
\gamma(i)_{\max }=10 \log _{10}\left(\frac{3}{\pi^{2}} \frac{10^{\frac{\Delta \gamma_{\max }}{10}}-1}{\left(\left(f_{c} \frac{v(i)}{c}+f_{o_{-} \max }\right) T\right)^{2}}\right)
$$

where the oscillator frequency offset $f_{o}(i)$ is replaced with a maximum tolerable offset $f_{o_{-} \max }$. Thus, the proposed scheme can adjust the transmit power with a maximum allowable power given as

$$
P_{X}(i)_{\max }=\frac{P_{N}}{|H(i)|^{2}}\left(\frac{3}{\pi^{2}} \frac{10^{\frac{\Delta \gamma_{\max }}{10}}-1}{\left(\left(f_{c} \frac{v(i)}{c}+f_{o_{-} \max }\right) T\right)^{2}}\right) .
$$

It implies that each user has its own maximum allowable transmit power, inversely proportional to its own channel gain and mobility. Thus, the users near the BS and/or the users in high mobility generally should reduce the transmit power (i.e., power adaptation) to keep the desired CIR loss, while the users with low mobility near the cell boundary do not need to reduce the transmit power, rather need to adjust the transmit rate for capacity improvement (i.e., rate adaptation).

\section{Performance evaluation}

The performance of the proposed scheme is verified by computer simulation. The system parameters and 
Table 1. OFDMA uplink system parameters

\begin{tabular}{|c|c|}
\hline Carrier frequency $\left(f_{c}\right)$ & $5.8 \mathrm{GHz}$ \\
\hline Bandwidth $(B)$ & $25 \mathrm{MHz}$ \\
\hline Symbol duration $(T)$ & $20.48 \mu \mathrm{sec}$ \\
\hline Number of subcarriers $\left(N_{c}\right)$ & 512 \\
\hline Tolerable oscillator frequency instability $\left(f_{o_{-} \max }\right)$ & $0.1 \mathrm{ppm}$ \\
\hline
\end{tabular}

Table 2. Simulation environments

\begin{tabular}{|c|c|c|c|}
\hline $\begin{array}{c}\text { Cell } \\
\text { configuration }\end{array}$ & \multicolumn{3}{|c|}{19 cells } \\
\hline Cell radius & \multicolumn{3}{|c|}{$1 \mathrm{~km}$} \\
\hline $\begin{array}{l}\text { Loading } \\
\text { factor }\end{array}$ & \multicolumn{3}{|c|}{$100 \%$} \\
\hline $\begin{array}{c}\text { Distribution } \\
\text { of user } \\
\text { location }\end{array}$ & \multicolumn{3}{|c|}{ Uniform distribution } \\
\hline \multirow{4}{*}{$\begin{array}{l}\text { Distribution } \\
\text { of user } \\
\text { mobility }\end{array}$} & & Type 1 & Type 2 \\
\hline & Pedestrian $(3 \mathrm{~km} / \mathrm{h})$ & $70 \%$ & $40 \%$ \\
\hline & Car $(60$ km/h) & $20 \%$ & $40 \%$ \\
\hline & $\begin{array}{l}\text { High speed railway } \\
(250 \mathrm{~km} / \mathrm{h})\end{array}$ & $10 \%$ & $20 \%$ \\
\hline $\begin{array}{c}\text { Link } \\
\text { adaptation }\end{array}$ & \multicolumn{3}{|c|}{ Ideal (i.e., using the Shannon's capacity curve) } \\
\hline
\end{tabular}

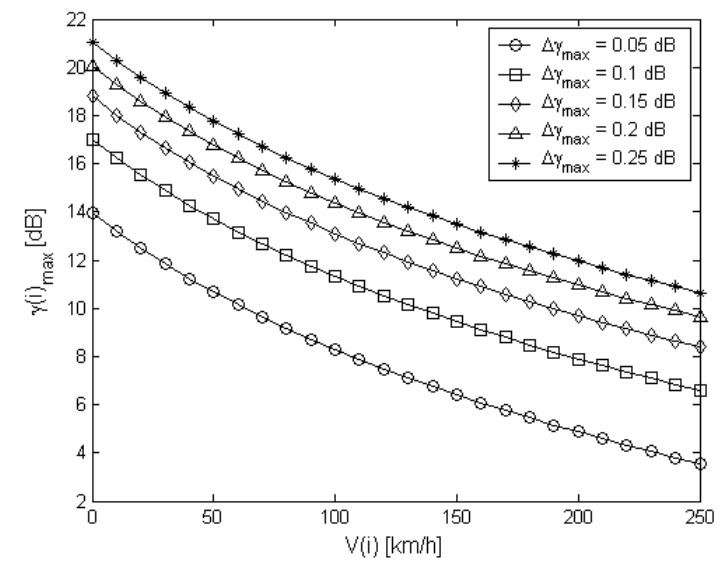

Figure 3. Maximum CIR due to the user mobility.

environments for the simulation are summarized in Table 1 and 2 [15].

Figure 3 depicts the maximum CIR in the presence of user mobility. Note that the maximum CIR without the user mobility is mainly limited by the frequency instability of oscillators. It can be seen that the users in low mobility environment have a loose restriction on the maximum CIR. For example, the user with $3 \mathrm{~km} / \mathrm{h}$ velocity has a maximum CIR of $13.73 \mathrm{~dB}$, while the user with $250 \mathrm{~km} / \mathrm{h}$ velocity has a maximum CIR of

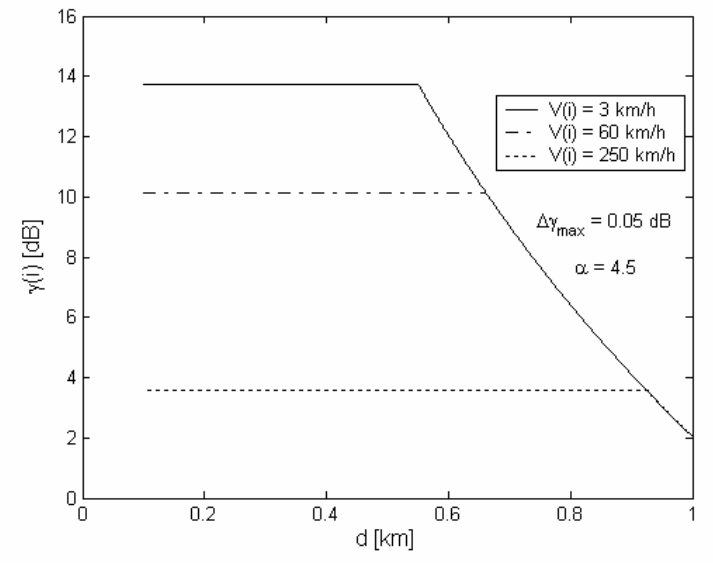

Figure 4. Adjusted CIR due to the distance.

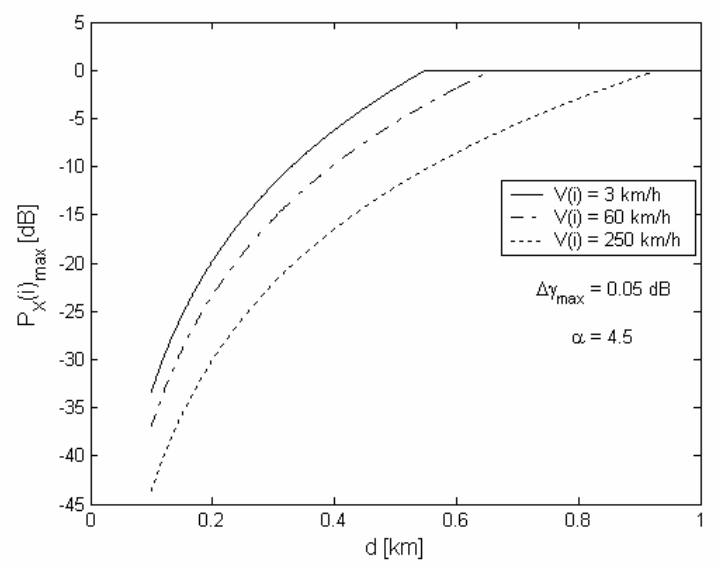

Figure 5. Power adjustment due to the distance.

$3.56 \mathrm{~dB}$ when $\Delta \gamma_{\max }=0.05 \mathrm{~dB}$. And it can also be seen that the restriction on the maximum $\mathrm{CIR}$ is proportional to $\Delta \gamma_{\max }$ which is pre-determined by the system requirement.

Figure 4 depicts the adjusted CIR due to the distance $d$ and Figure 5 depicts the corresponding power adjustment when the path loss exponent $\alpha$ is 4.5 and $\Delta \gamma_{\text {max }}=0.05 \mathrm{~dB}$. It can be seen that the user near the cell center needs to reduce the transmit power as shown in Figure 5 to keep the CIR below the desired maximum CIR, while the user near the cell boundary does not need to adjust the transmit power, rather needs to adjust the transmit rate. For example, the user with $3 \mathrm{~km} / \mathrm{h}$ velocity within $0.55 \mathrm{~km}$ radius needs to reduce the transmit power. However, the user outside $0.55 \mathrm{~km}$ radius does not need to adjust the transmit power, rather it needs to adjust the transmit rate. It can also be seen that the users in the higher mobility environment should transmit the signal with reduced transmit power at the same distance from the BS due to 


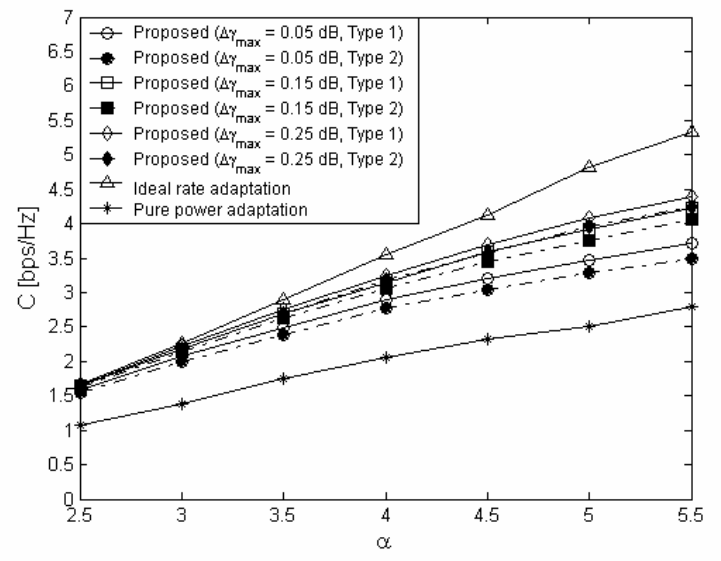

Figure 6. System capacity of the proposed scheme.

the ICI. For example, the user with $3 \mathrm{~km} / \mathrm{h}$ velocity does not need to reduce the transmit power (only need to adjust the rate), while the user with $250 \mathrm{~km} / \mathrm{h}$ velocity should reduce the transmit power by $8.6 \mathrm{~dB}$ when is apart by $0.6 \mathrm{~km}$ distance from the BS. Thus the users in low mobility environment have a large rate adaptation region and can achieve a higher capacity.

Figure 6 depicts the system capacity of the proposed scheme for various path loss exponents, allowable CIR loss limit and user mobility in Table 2 . We consider two operating conditions, type 1 and 2 model, representing a low and high mobility environment, respectively. The type 1 model comprises users with $70 \%$ pedestrians of $3 \mathrm{~km} / \mathrm{h}$ velocity, $20 \%$ cars of 60 $\mathrm{km} / \mathrm{h}$ velocity and $10 \%$ high speed railway of 250 $\mathrm{km} / \mathrm{h}$ velocity, while the type 2 model comprises users with $40 \%$ pedestrians, $40 \%$ cars and $20 \%$ high speed railways. As an upper bound of the performance, we also depict the performance of ideal rate adaptation without ICI, referred to "Ideal rate adaptation", although it is not practical at all. We also depict the performance of pure power adaptation scheme. It can be seen that the proposed scheme outperforms the pure power adaptation scheme by more than $0.5 \mathrm{bps} / \mathrm{Hz}$ and shows superiority in low mobility environments. And the proposed scheme can increase the system capacity by loosing the CIR loss condition (i.e., increasing $\left.\Delta \gamma_{\max }\right)$.

\section{Conclusion}

In this paper, we have considered the ICI problem in the uplink of OFDMA-based cellular systems. To alleviate the ICI problem, we have proposed hybrid use of power and rate adaptation. The proposed scheme adapts the transmit power to limit the ICI to a desired level and adjusts the transmit rate to achieve high capacity. Thus, it can improve the system capacity compared to the use of pure power adaptation, while guaranteeing a desired maximum CIR loss. Finally, the performance of the proposed scheme has been verified by computer simulation in various OFDMA uplink environments. The simulation results show that the proposed scheme outperforms conventional pure power adaptation schemes.

\section{References}

[1] K. Baum, T. Kostas, P. Sartori and B. Classon, "Performance characteristics of cellular systems with different link adaptation strategies," IEEE Trans. Veh. Tech., vol. 52, pp. 1497-1507, Nov. 2003.

[2] J. Zander, "Performance of optimum transmitter power control in cellular radio systems," IEEE Trans. Veh. Tech., vol. 41, pp. 57-62, Feb. 1992.

[3] G. Foschini and Z. Miljanic, "A simple distributed autonomous power control algorithm and its convergence," IEEE Trans. Veh. Tech., vol. 42, pp. 641-646, Nov. 1993.

[4] W. T. Webb and R. Steele, "Variable rate QAM for mobile radio," IEEE Trans. Commun., vol. 43, pp. 22232230, July 1995.

[5] H. Matsuoka, S. Sampei, N. Morinaga and Y. Kamio, "Adaptive modulation system with variable coding rate concatenated code for high quality multi-media communication systems," in Proc. IEEE VTC'96, pp. 487491, Apr. 1996.

[6] J. M. Torrance and L. Hanzo, "Upper bound performance of adaptive modulation in a slow Rayleigh fading channel," Electron. Lett., vol. 32, no. 8, pp. 718-719, Apr. 1996.

[7] R. V. Nee and R. Prasad, OFDM for wireless multimedia communications, Altech House, 2000.

[8] S. Hara and R. Prasad, "Overview of multicarrier CDMA,” IEEE Commun. Mag., pp. 126-133, Dec. 1997.

[9] J. A. C. Bingham, "Multicarrier modulation for data transmission: An idea whose time has come," IEEE Commun. Mag., vol. 28, pp5-14, May 1990.

[10] Flarion, The benefits of a packet-switched, ALL-IP mobile broadband network, Flarion white paper (http://www.flarion.com), Feb. 2004.

[11] IEEE P802.16e, Draft IEEE standard for local and metropolitan area networks, Sept. 2004.

[12] P. Robertson and S. Kaiser, "Analysis of the loss of orthogonality through Doppler spread in OFDM systems," in Proc. IEEE Globecom'99, pp. 701-706, Dec. 1999.

[13] Wang, J., and Ng, T. S., Advances in $3 G$ Enhanced Technologies for Wireless Communications, Artech House, 2002.

[14] A. Hutter, "Design of OFDM systems for frequencyselective and time-variant channels," in Proc. IEEE IZS'02, Feb. 2002.

[15] J. Moon, J.-Y. Ko and Y.-H. Lee, "A framework design for the next-generation radio access system," IEEE J. Select. Areas Commun., vol. 24, no. 3, pp. 554-564, Mar. 2006. 